\title{
A Flow Time Model for Melt-Cast Insensitive Explosive Process
}

\author{
Guillemin Jean-Philippe*, Brunet Luc \\ GIAT industries, 7 route de Guerry, 18023 Bourges Cedex (France)
}

Bonnefoy Olivier, Thomas Gérard

Ecole Nationale Supérieure des Mines de Saint-Étienne, Centre SPIN/LPMG, UMR CNRS 5148, 158 Cours

Fauriel, 42023 Saint-Étienne Cedex 2 (France)

\begin{abstract}
Diphasic flows of concentrated suspensions of melt-cast insensitive explosives exhibit particular rheology properties. In order to limit the handling of pyrotechnical products presenting a risk with respect to the mechanical and thermal shocks, a lot of works have been undertaken for many years in the civil engineering sector. The objective of this study is to propose a predictive model of the flow time of a concentrated suspension through a nozzle located at the bottom of a tank. Similarly to our industrial process, the suspension is made out of insensitive energetic materials and flows under gravity. Experimental results are compared to three models (Quemada, Krieger-Dougherty, and Mooney) predicting the viscosity $\mu$ of a suspension as a function of the solid volume fraction $\phi$, the maximum packing density $\phi_{m}$ and the viscosity $\mu_{0}$ of the interstitial liquid. De Larrard's model is used to calculate $\phi_{m}$. The value of viscosity measured for the pure liquid is near the one predicted by the Bernoulli theorem, where liquids are considered as incompressible and inviscid. In the end, it turns out that the Quemada's model gives a fair agreement between predictions and experiments.
\end{abstract}

Keywords: Melt-Cast Insensitive Explosive, Rheology of Concentrated Suspensions, Flow Time Model, Maximum Packing Density, Quemada's Model for Viscosity, De Larrard's Model,

\section{Introduction}

The purpose of this article is to propose a predictive model of the flow time necessary for emptying a reactor filled with a concentrated suspension through a nozzle situated at the bottom. Similarly to our industrial process, the suspension is made out of insensitive energetic materials and flows under gravity. Experimental results are compared with three viscosity models largely used in the field of concentrated suspensions (Quemada [1], Krieger and Dougherty [2] and Mooney [3]). These models give the viscosity of a concentrated suspension as a function of the viscosity $\mu_{0}$ of the pure liquid, the volume fraction $\phi$ of the solid included in the paste, and the maximum packing density $\phi_{m}$.

* Corresponding author: email: jp.guillemin@giat-industries.fr
These parameters will be used as a basis to establish our model. First, a relationship between the viscous and flow time terms starting from Navier-Stokes equation is needed. Secondly, evaluations of some characteristics of the granular phase in concentrated suspensions will be obtained and inserted in our flow time equations. De Larrard's model for the calculation of the maximum packing density $\phi_{m}$ [4] will be considered. The study of the dependence of the casting time upon weak variations of the formulation and upon the choice of raw materials will make possible to compare the experimental results and those given by our model. Melt casting energetic formulations are established for use in a cylindrical tank of a total volume of $5700 \mathrm{~cm}^{3}$. The experiments for flow time determinations are all performed in this container.

\section{Model description}

\subsection{Rheology of Concentrated Suspensions}

Since the publication of Einstein analysing the viscosity of dilute suspensions of rigid spheres in a viscous liquid, numerous equations have been developed to try and extend Einstein's formula to suspensions of higher concentrations [5]. The various resulting formulas differ considerably from each other. In this study, three relationships have been selected to express the viscosity of dispersions of spherical particles as a function of the viscosity of the interstitial fluid $\mu_{0}$, the volume fraction of solids $\phi$ and the maximum packing density of the solids $\phi_{m}$. Others equations are given in the literature, but the three retained hereafter seem to be the most encountered, discussed and reliable, and this is the reason why they have been chosen in this study. These equations are given in Tab. 1 . 
Table 1. Rheological equations

\begin{tabular}{cc}
\hline Authors & Rheological equations \\
\hline Krieger-Dougherty [2] & $\mu=\mu_{0}\left(1-\frac{\phi}{\phi_{m}}\right)^{-2.5 \phi_{m}}$ \\
Quemada [1] & $\mu=\mu_{0}\left(1-\frac{\phi}{\phi_{m}}\right)^{-2}$ \\
Mooney [3] & $\mu=\mu_{0} \exp \left(\frac{2.5 \phi}{1-\frac{\phi}{\phi_{m}}}\right)$ \\
\hline
\end{tabular}

\section{$2.2 \quad$ Model}

\subsubsection{Relationship between Viscosity and Flow \\ Time}

Because of the high solid content and the low flow velocity, it is assumed here that Reynolds number is not too important, although greater than unity. The inertial term of the Navier-Stokes equation is supposed to be small enough with respect to the viscous term. The momentum equation takes the following simplified form:

$$
\rho \cdot \frac{\partial \vec{v}}{\partial t}=-\vec{\nabla} p+\mu \cdot \Delta \vec{v}+\rho \cdot \vec{g}
$$

As the suspension flows during a relatively long time (over one minute), the state can be regarded as quasi-stationary and one can write at any time:

$$
\mu . \Delta \vec{v}=\vec{\nabla} p-\rho \cdot \vec{g}
$$

Eq. (2) implies that, for a given pressure gradient, the flow rate of a fluid is inversely proportional to its viscosity. This can be applied to the two fluids considered, the suspension with a viscosity $\mu$ and the interstitial fluid with a viscosity $\mu_{0}$ Therefore, for a given volume, the flow time $\tau$ is directly proportional to the viscosity:

$$
\frac{\tau}{\tau_{0}}=\frac{\mu}{\mu_{0}}
$$

Where $\tau_{0}$ represents the flow time for the interstitial fluid.

\subsubsection{Calculation of $\tau_{0}$ by Bernoulli's approach}

The interstitial fluid is a mixture of trinitrotoluene TNT and additives, presenting viscosities of 11 and $48.6 \mathrm{mPa}$.s respectively. Such values are rather small, and the interstitial fluid can be assimilated to a perfect fluid: this allows us to use the Bernoulli relationship in the tank described on Fig. 1.

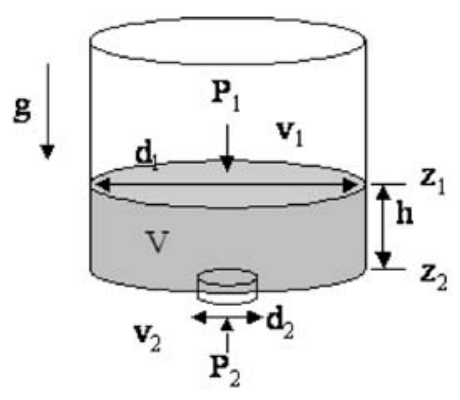

Figure 1. Tank geometry

Where $V\left[\mathrm{~m}^{3}\right]$ is the suspension volume; $v_{1}$ and $v_{2}$ $[\mathrm{m} / \mathrm{s}]$ are the fluid velocities; $P_{1}$ and $P_{2}[\mathrm{~Pa}]$ are the top and bottom pressures; $d_{1}$ and $d_{2}[\mathrm{~m}]$ are the diameters of sections 1 and $2 ; h=z_{1}-z_{2}$ [m] is the fluid height; $g\left[\mathrm{~m} / \mathrm{s}^{2}\right]$ is the acceleration of gravity equal to $9.81 \mathrm{~m} / \mathrm{s}^{2}$.

The Bernoulli relationship can be written:

$\frac{\rho v_{1}^{2}}{2}+P_{1}+\rho g z_{1}=\frac{\rho v_{2}^{2}}{2}+P_{2}+\rho g z_{2}$

Where $\rho$ represents the fluid density $\left[\mathrm{kg} / \mathrm{m}^{3}\right]$.

The mass balance gives:

$\rho v_{1} d_{1}^{2}=\rho v_{2} d_{2}^{2}$

With $v_{1}=-d h / d t$, so that Eq. (4) could be written now:

$\frac{d h}{d t}=\sqrt{\left(\frac{a \cdot(h+b)}{c}\right)}$

Where $a=2 g ; b=\left(P_{1}-P_{2}\right) /(\rho g) ; c=\left(d_{1} / d_{2}\right)^{4}-1$

The integration of Eq. (6) between $\mathrm{t}=0$ and $t=\tau$, where the liquid heights are respectively $h_{0}$ and $h(\tau)$ leads to Eq.7:

$\sqrt{h(\tau)+b}=\sqrt{h_{0}+b}-\tau \sqrt{\frac{a}{4 . c}}$

At $\tau_{0}$, the tank is empty and $h\left(\tau_{0}\right)=0$. Finally, $\tau_{0}$ is given by Eq. (8)

$\tau_{0}=\sqrt{\left(\frac{d_{1}}{d_{2}}\right)^{4}-1} \cdot \sqrt{\left(\frac{2}{g}\right)} \cdot\left[\sqrt{h_{0}+\frac{\left(P_{1}-P_{2}\right)}{\rho g}}-\sqrt{\frac{\left(P_{1}-P_{2}\right)}{\rho g}}\right]$

With Eq. (3), Eq. (8) and one of the rheological equations given in Tab. 1, three relationships, Eq. (9), Eq. (10) and Eq. (11), allow us to calculate the flow time for our energetic concentrated suspensions. These relationships depend on the solid volume fraction $\phi$, the flow for the interstitial fluid $\tau_{0}$ and the maximum packing density $\phi_{m}$. 


$$
\begin{aligned}
& \tau=\tau_{0}\left(1-\frac{\phi}{\phi_{m}}\right)^{-2.5 \phi_{m}} \\
& \tau=\tau_{0}\left(1-\frac{\phi}{\phi_{m}}\right)^{-2} \\
& \tau=\tau_{0} \exp \left(\frac{2.5 \phi}{1-\frac{\phi}{\phi_{m}}}\right)
\end{aligned}
$$

\subsubsection{Calculation of the Maximum Packing Density}

Many studies aim at improving predictions of viscosity by refining the methods of calculation of the maximum packing density [6-7-8]. Many strong similarities appear between concrete and explosive made by casting process as the great number of granulometric scales, and the range of the component morphologies. In this kind of approach, the model developed by De Larrard seems very attractive [9]. This model predicts the maximum packing density of a polydisperse mix, from three parameters: the particle size distribution of the mix, its true density and the experimental packing density of the solid species. The use of software is required to determine the maximum packing density. In this study, the RENE-LCPC software developed by De Larrard and T. Sedran [10] has been selected. The details of the algorithm will not be described here but the interested reader may refer to some of the associated publications [11-1213].

This model deals with grain mixtures in which linear combinations of packing densities allow to predict the packing density of a mixture of monosized particles $d_{i}\left(d_{1}<d_{2}<\ldots<d_{n}\right)$ from:

$$
\phi_{i}=\frac{\beta_{i}}{1-\left(1-\beta_{i}\right) \sum_{j=1}^{i-1} a_{i j} \alpha_{i}-\sum_{j=i+1}^{N} b_{i j} \alpha_{j}}
$$

Where $\phi_{i}$ is the packing density of class; $\alpha_{i}$ is the volume fraction of belonging to class $i ; \beta_{i}$ is the residual packing density i.e. when the class $i$ is alone and fully packed. To compute the packing density of the overall mixture, one considers that the bulk volume of the class $i$ fills the porous space around the coarser grains; moreover, the volume of finer classes inserted in the voids of class $i$ must be added. Two interaction effects must be taken into account in this calculation: the wall effect, $a_{i j}$, exerted by the coarser particles and the loosening effect, $b_{i j}$, exerted by the finer particles. Finally, $\phi_{m}$ is given by Eq. (13).

$$
\phi_{m}=\min _{1<i<N} \phi_{i}
$$

\section{Raw Materials and Experimental Set-up}

\begin{tabular}{|c|c|c|c|c|}
\hline Compounds & Name & $\begin{array}{c}\text { True Density } \\
\left(\mathrm{g}\left(\mathrm{cm}^{3}\right)\right. \\
\end{array}$ & $\begin{array}{c}\text { Melting Point } \\
\left({ }^{\circ} \mathrm{C}\right) \\
\end{array}$ & Physical state at exp. Conditions \\
\hline TNT & 2,4,6-trinitrotoluene & 1.65 & 81 & liquid \\
\hline A & Additive & 1 & 83 & liquid \\
\hline $\mathrm{Al}$ & Aluminium & 2.7 & 660 & solid \\
\hline NTO & 3-nititro-1,2,4-triazol-5-one & 1.92 & 279 & solid \\
\hline
\end{tabular}

\subsection{Raw Materials}

The compounds used in this study are listed in Tab. 2.

Table 2. Studied compounds

\subsubsection{Rheology of the Liquid Phase}

The liquid phase is composed of TNT and a fusible additive A. The Newtonian viscosity of the additive is measured by a viscometer presenting a"Couette" geometry (Rheomat 30, Contrave). A thermo-regulated bath is used to control the temperature. Results show that the additive has a Newtonian behaviour and its Newtonian viscosity is equal to $48.6 \mathrm{mPa}$.s at $85^{\circ} \mathrm{C}$. The TNT Newtonian viscosity $\mu_{T N T}$ is calculated with Eq. (14) established by [14] on temperature as follows:

$\mu_{T N T}=\mu^{*} \exp \left(\frac{Q}{T}\right)$

Where $\mathrm{T}$ is the absolute temperature in Kelvin and $\mu^{*}=5.41 \cdot 10^{-7}$ Pa.s and $Q=3570 \mathrm{~K}$.

With these parameter values, $\mu_{T N T}=11 \mathrm{mPa} . \mathrm{s}$ at $85^{\circ} \mathrm{C}$.

\subsubsection{Solid Phase Characterization}

The solid phase is made out of two species: NTO and aluminium. Four batches of NTO, labelled NTO 1, NTO 2, NTO 3, NTO 4 and two batches of aluminium, Al 1, Al 2 are used. They differ by their packing densities, grain size distributions and morphologies.

The experimental packing density $C$ is calculated from the true and bulk densities. The true density $\rho_{t}$ is measured with a helium pycnometer from Micromeritics and the true bulk density $\rho_{b}$ with a volumenometer. $C$ is defined by Eq. (15):

$C=\frac{\rho_{t}}{\rho_{b}}$

The particle size distribution is determined by sifting under vibrations NTO particles, and for aluminium particles with a laser granulometer 
(Malvern Mastersizer 2000). Grain morphologies are determined by scanning electron microscopy. Average sizes of NTO particles reach $350-400 \mu \mathrm{m}$ and $13 \mu \mathrm{m}$ for aluminium particles. SEM analysis shows spherical morphology for Al 2 .

Table 3. NTO and Al experimental packing densities

\begin{tabular}{cccc}
\hline Batches & $\rho_{t}\left(\mathrm{~g} / \mathrm{cm}^{3}\right)$ & $\rho_{b}\left(\mathrm{~g} / \mathrm{cm}^{3}\right)$ & $C$ \\
\hline NT0 1 & 1.92 & 0.99 & 0.52 \\
NT02 & 1.92 & 0.97 & 0.51 \\
NT03 & 1.92 & 0.75 & 0.39 \\
NT0 4 & 1.92 & 0.79 & 0.41 \\
Al 1 & 2.7 & 1.1 & 0.41 \\
Al2 & 2.7 & 1.2 & 0.44 \\
\hline
\end{tabular}

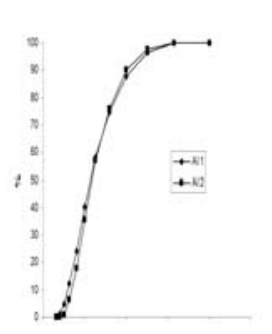

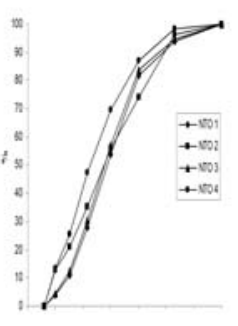

$d(\mathbf{x})$
Figure 2. $\mathrm{Al}$ and NTO particle size distributions

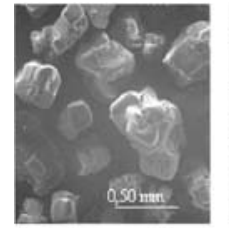

NTO 1

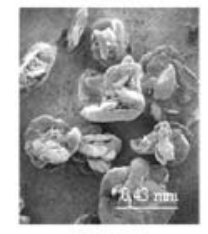

NTO 3

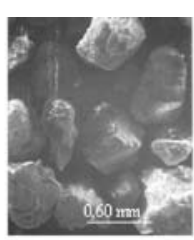

NTO 2

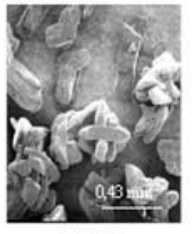

NTO 4

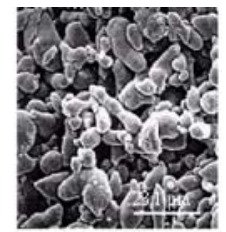

Al 1

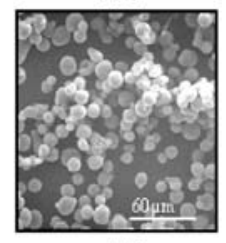

Al 2
Figure 3. NTO and $\mathrm{Al}$ morphologies

\subsection{Experimental Set-Up}

Nine insensitive explosive formulations are elaborated with different volume fractions of raw materials. These formulations are given in the left hand side of Tab. 4. The flow time of $5700 \mathrm{~cm}^{3}$ of each suspension has been measured and the texture of these energetic pastes has been observed. For the energetic formulations exhibiting a high apparent viscosity, the measurements of the flow time has been made several times to get significant results. The measurement precision decreases when explosive formulations are highly concentrated. In this case, we repeated twice the measurement of the flow time.

As in our industrial process, suspensions flow through a nozzle situated at the bottom of a tank under gravity. The dimensions of the cylindrical tanks are represented on Fig. 4.
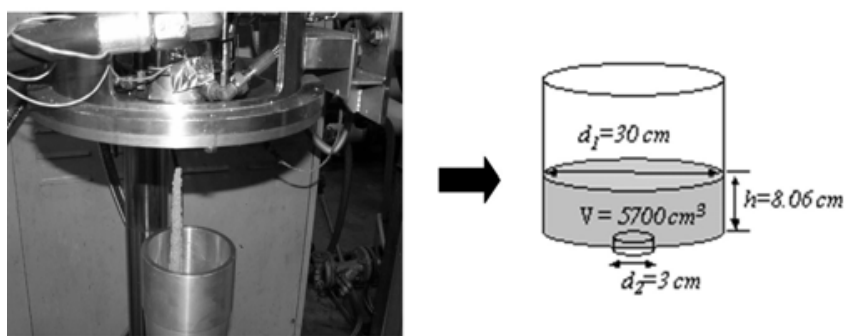

Figure 4. Left: Industrial process; Right, scheme of the tank with some key dimensions

\section{Results and Discussion}

\subsection{Experimental Results}

The flow time and texture of each formulation is reported in the right hand side of Tab. 4 and depicted on Fig. 5. The last formulation (Formulation 9) represents the interstitial fluid, composed of TNT and additive only.

Table 4. Composition and flow time of each insensitive energetic formulation

\begin{tabular}{c|cc|cccccc}
\hline \multirow{2}{*}{ Formulation } & \multicolumn{2}{c}{ Batche } & \multicolumn{5}{c}{ Volume Fraction $\phi_{i}$} \\
& Al & NT0 & TNT & A & Al & NTO & $\tau(s)$ \\
\hline 1 & 1 & 2 & 0.33 & 0.13 & 0.09 & 0.45 & 72 \\
2 & 1 & 1 & 0.33 & 0.13 & 0.09 & 0.45 & 168 \\
3 & 1 & 3 & 0.33 & 0.13 & 0.09 & 0.45 & 720 \\
4 & 1 & 3 & 0.33 & 0.13 & 0.09 & 0.45 & 1200 \\
5 & 2 & 2 & 0.33 & 0.13 & 0.09 & 0.45 & 64 \\
6 & 1 & 4 & 0.35 & 0.14 & 0.07 & 0.44 & 330 \\
7 & 1 & 4 & 0.35 & 0.14 & 0.07 & 0.44 & 303 \\
8 & 2 & 4 & 0.35 & 0.14 & 0.07 & 0.44 & 220 \\
9 & NA & N/A & 0.72 & 0.28 & 0 & 0 & 11 \\
\hline
\end{tabular}

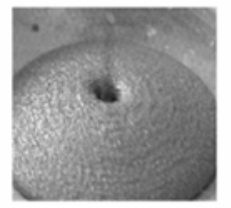

Fonnulation 1

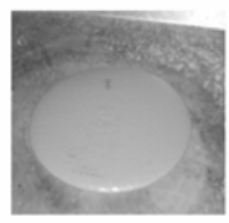

Formulation $S$

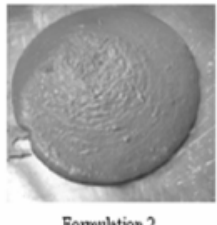

Formulation 2

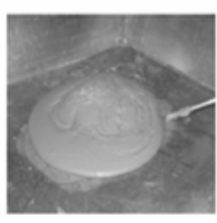

Fomolation 6 and ?

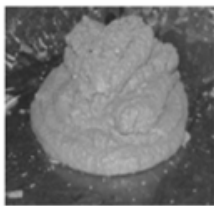

Formulation 3 and 4

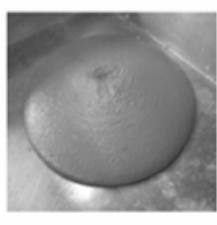

Formulation 8
Figure 5. Texture of insensitive energetic pastes

\subsection{Comparison between Experimental and Modelling Results}

The maximum packing density $\phi_{m}$ and the ratio between the maximum packing density and the solid volume fraction $\phi$ are given in the left hand side of Tab. 5 . 
Theoretical flow times are calculated Eq. (9), Eq. (10) and Eq. (11). Final results are listed in the right hand side of Tab. 5. Results from Eq. (10), which are a direct application of Quemeda's model, are in agreement with our experimental results.

Table 5. Maximum packing density of each formulation and comparison between theoretical $\left(\tau_{\text {theo }}\right)$ and experimental results $\left(\tau_{\text {exp }}\right)$

\begin{tabular}{|c|c|c|c|c|c|c|}
\hline Formulation & $\phi_{m}$ & $\phi / \phi_{m}$ & \multicolumn{3}{|c|}{$\tau_{\text {theo }}(\mathrm{s})$} & $\tau_{\exp }(\mathrm{s})$ \\
\hline 1 & \begin{tabular}{|l|l|}
0,79 \\
\end{tabular} & 0,68 & 128 & 124 & 912 & 72 \\
\hline 2 & 0,79 & 0,68 & 128 & 124 & 912 & 168 \\
\hline 3 & 0,61 & 0,89 & 972 & 348 & 1645790 & 720 \\
\hline 4 & 0,61 & 0,89 & 972 & 348 & 1645790 & 1200 \\
\hline 5 & 0,79 & 0,68 & 121 & 121 & 815 & 64 \\
\hline 6 & 0,63 & 0,81 & 353 & 174 & 10334 & 330 \\
\hline 7 & 0,63 & 0,81 & 353 & 174 & 10334 & 303 \\
\hline 8 & 0,63 & 0,81 & 353 & 174 & 10334 & 220 \\
\hline 9 & 0 & 0 & 13 & 13 & 13 & 11 \\
\hline & & $\mathrm{R}^{2}$ & 0,942 & 0,914 & 0,910 & \\
\hline
\end{tabular}

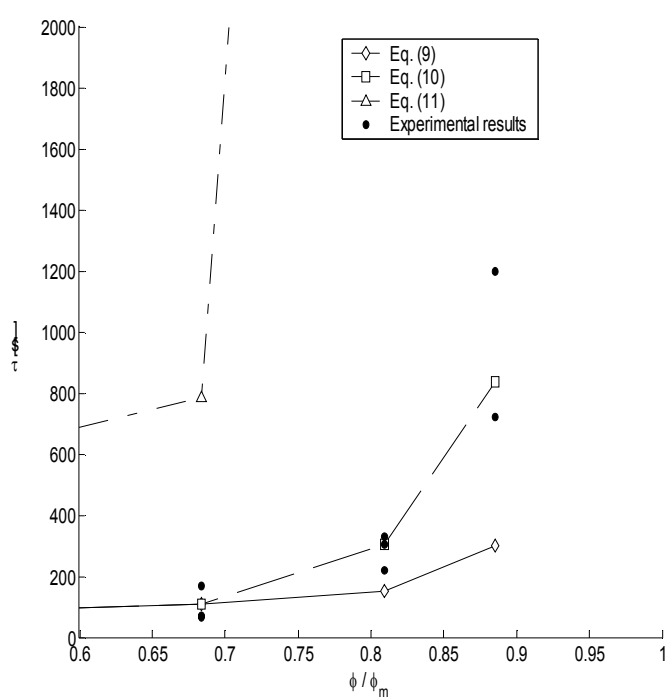

Figure 6. Comparison between theoretical and experimental results

\subsection{Discussion}

We assumed that the interstitial fluid can be considered as inviscid; this seems to be quite realistic since the experimental flow time $(11 \mathrm{~s})$ for $5700 \mathrm{~cm}^{3}$ corresponds to the theoretical time (13 s) calculated by Eq. (8). Moreover, the fair agreement between our experimental results for concentrated suspensions and the theoretical predictions from Quemada's model seems to support our second, intuitive, assumption that the loss of energy due to viscous effects remains negligible. The ratio of the maximum packing density to the solid volume fraction (left hand side of $\mathrm{Tab} 5$ ) gives an interesting information to be compared with the texture of insensitive energetic pastes (Fig 5). When it is close to 1 (Formulations 3 and 4), i.e. near the jamming of the structure, the texture is very pasty. On the opposite side, when $\phi / \phi_{m}$ decreases
(Formulations 1, 2 and 5) or far from 1, energetic pastes present a more liquid behaviour.

As shown on Fig. 6, theoretical values of the flow time resulting from Eq. (9) and Eq. (11) show significant deviations from experimental results. We find that Eq. (10) derived from Quemada's model reflects our experimental results more nicely.

Overall, this result completely ignores the nonNewtonian nature of these energetic suspensions. Typically, energetic suspensions exhibit yield stresses, wall slip and sensitivity to shearing stresses. For more details of the viscoplastic behaviour of concentrated energetic materials, the reader will be able to refer to last work of Kalyon [15-16].

\section{Conclusions}

For suspensions of different formulations, various experiments of casting under gravity have been carried out and the time required by a given volume to pass through a nozzle has been measured.

Considering that the suspension flow proceeds under conditions where the inertial term of the Navier-Stokes equation is negligible compared to the viscous term, we find the suspension flow time to be directly proportional to its viscosity. Consequently, the cast time can be calculated from the suspension viscosity. Three models, frequently used in rheology, make possible to calculate this viscosity as a function of the interstitial fluid Newtonian viscosity and the ratio $\phi / \phi_{m}$ where $\phi_{m}$ is calculated with De Larrard's model. The relatively low viscosity of the interstitial fluid allows us to consider perfect fluids and apply the Bernoulli relationship. Comparison between theoretical values and experimental results shows that Quemada's model gives a satisfactory modelling. In the configuration studied, the flow time can be estimated by the following relationship:

$$
\tau=\tau_{0} \cdot\left(1-\frac{\phi}{\phi_{m}}\right)^{-2}
$$

\section{References}

[1] D. Quemada, Rheology of Concentratred Disperse Systems and Minimum Energy Dissipation Principle, I : Viscosity-Concentration Relationship, Rheol. Acta, 1977, 16, 82.

[2] I.M. Krieger, T.J. Dougherty, A mechanism for non-Newtonien flow in suspensions of rigid spheres, Trans. Soc. Rheol., 1959, 3, 137.

[3] M. Mooney, The Viscosity of a Concentrated Suspension of Spherical Particles, J. Colloid Sci., 1951, 6, 162.

[4] B. Bournonville, P. Coussot, X. Château, Modification du modèle de Farris pour la prise en compte des interactions géométriques d'un 
mélange polydisperse de particules, Rhéologie. 2005, 7, 1-8.

[5] U. Teipel, Energetic Materials, Particle Processing and Charactezation, WILEY-VCH 2005, p. 443.

[6] Z Toutou,. C. Lanos, Y. Melingue, N. Roussel, Modèle de Viscosité Multi-échelle : de la Pâte de Ciment au Micro-béton, Rhéologie. 2004, 5, 1.

[7] M. Gan, N. Gopinathan, X. Jia and R.A. Williams, Predicting Packing Characterisics of Particles of Arbitrary Shapes, KONA, 2004, 22, 82.

[8] P. Stroeven, M. Stroeven, Dynamic Computer Simulation of Concrete on Different Levels of the Microstructure- Part 1, Image Anal. Stereol. 2003, 22,1 .

[9] F. De Larrard, T. Sedran, Mixture-Proportioning of High-Performance Concrete, Cement and Concrete Research. 2002, 32, 1699

[10] T. Sedran, F. De Larrard, René-LCPC, Un Logiciel pour Optimiser la Granularité des Matériaux de Génie Civil, Note Technique $\mathrm{N}^{\circ} 194$, 1994. Laboratoire des Ponts et Chaussées, France.

[11] T.Stovall, F. De Larrard, M. Buil, Linear Packing Density Model of Grain Mixtures, Powder Technol., 1986, 48, 1 .

[12] F. De Larrard, Formulation et Propriétés des Bétons à très Hautes Performances, Rapport des Laboratoires des Ponts et Chaussées $N^{\circ} 149$, 1967. Thèse de Doctorat de l'Ecole Nationale des Ponts et Chaussées, France.

[13] F. De Larrard, A General model for the prediction of voids content in high performance concrete mix design, CANMET/ACI Conference on Advances in Concrete Technology, Athenes, 1992.

[14] M.A Parry, H.H. Billon, A Note on the Coefficient of viscosity of pure molten 2,4,6 trinitrotoluene (TNT), Rheol Acta. 1988, 28, 661.

[15] D. Kalyon, An overview of the rheological behaviour and characterization of energetic formulations: ramifications on safety and products quality, Journal of Energetic Materials. 2006, 24, 213-245.

[16] D. Kalyon, Apparent slip and viscoplasticity of concentrated suspensions, J. Rheology. 2005, 49, 3, 621-640.

\section{Acknowledgments}

The authors thank L. Chouris as the Person in Charge of the realization of the tests.

Symbols and Abbreviations

$\begin{array}{ll}P & \text { Pressure }(\mathrm{Pa}) \\ v & \text { Velocity }(\mathrm{m} / \mathrm{s}) \\ h & \text { Fluid height }(\mathrm{m}) \\ V & \text { Volume of suspension }\left(\mathrm{m}^{3}\right) \\ g & \text { Acceleration of gravity }\left(\mathrm{m} / \mathrm{s}^{2}\right) \\ C & \text { Experimental packing density } \\ \rho_{t} & \text { True density }\left(\mathrm{g} / \mathrm{cm}^{3}\right) \\ \rho_{b} & \text { Bulk density }\left(\mathrm{g} / \mathrm{cm}^{3}\right)\end{array}$

$\begin{array}{ll}\mu & \begin{array}{l}\text { Viscosity of suspensions } \\ \text { (Pa.s) }\end{array} \\ \mu_{0} & \text { Newtonian viscosity of } \\ & \text { interstitial fluid (Pa.s) } \\ \tau & \text { Flow time of suspension (s) } \\ \tau_{0} & \text { Flow time of interstitial fluid } \\ & (\mathrm{s}) \\ \phi & \text { Solid Volume fraction (-) } \\ \phi_{m} & \text { Maximum packing density (-) } \\ \phi_{i} & \text { Packing density of class } i(-) \\ \beta_{i} & \text { Residual packing density of class } \\ a_{i j} & i(-) \\ b_{i j} & \text { Wall effect coefficient (-) } \\ T & \text { Loosening effect coefficient (-) }\end{array}$

Viscosity of suspensions

Newtonian viscosity of

nterstitial fluid (Pa.s)

Flow time of interstitial fluid

Solid Volume fraction (-)

Maximum packing density (-) 